\title{
Unsteady Transonic Flows with Shock Waves in an Asymmetric Channel
}

\author{
John S.-K. Chan * and T. C. Adamson Jr. $\dagger$ \\ The University of Michigan, Ann Arbor, Mich.
}

\begin{abstract}
Two-dimensional inviscid, unsteady transonic flows with shock waves in asymmetric channels having arbitrary wall shapes are investigated, using the method of matched asymptotic expansions. With the exception of thin regions in the neighborhood of both the channel throat and the shock wave, solutions found using inearized governing equations are valid. In the region enclosing the shock wave, an inner solution, which satisfies the shock jump conditions and matches with the outer solutions, is presented. The shock shape is found as part of the solution, which is obtained numerically using the method of integral relations. A composite solution, uniformly valid throughout the channel, and the relation between the instantaneons shock wave position and back pressure far downstream are presented.
\end{abstract}

\section{Introduction}

$\mathrm{T}$ HE analysis of two-dimensional transonic channel flows with shock waves has recently been the subject of several papers, ${ }^{1-6}$ culminating in a description of solutions found for insteady flows with arbitrary initial conditions and wall shapes. ${ }^{6}$ However, the solutions, no matter whether the channels are symmetric or asymmetric, are valid for streamine curvatures small enough that, to the scale of the channel width, the shock waves are planar. In this paper, on the other hand, a lower order (larger) curvature is considered in an asymmetric channel, so that strong cross-channel gradients in velocity and pressure are found in the lowest order solutions. As a result, the shock wave is not planar; its shape is unknown and must be found as part of the solution.

Two approaches have generally been used in constructing analytical solutions for transonic channel flows with shock waves. In one, similarity solutions of the transonic smalldisturbance equation are sought through various transCormations, resulting in solutions for steady ${ }^{1}$ or unsteady ${ }^{2}$ flows. The solutions thus obtained, however, satisfy only very special bondary conditions, which may or may not correspond to the physical problem under consideration, and the solutions are valid in a thin neighborhood of the sonic line. In the other approach, the method of matched asymptotic expansions is used to derive governing equations for each order of approximation from the general inviscid flow equations for various regions in the channel. Adamson, Messiter, and Richey ${ }^{4}$ showed that, in the main part of the channel, this method gives a systematic derivation of the power series expansion solution postulated by Szaniawski and used, for example, in the paper by Kopystynski and Szaniawski. ${ }^{3}$ In this method, arbitrary wall shapes may be specificd, and in the extension to unsteady flows, ${ }^{6}$ arbitrary ininal conditions may be considered. It is this approach which is used in the present paper.

Th the following, the flow is taken to be two-dimensional, compressible, and transonic. The gas is assumed to follow the perfect gas law and have constant specific heats. Upstream of the shock wave the flow is considered to be irrotational, and the Reynolds number is taken to be large enough that viscous effects are negligible. The unsteadiness in the flow is assumed to arise as a result of disturbances impressed upon the flow

Received May 23, 1977. Copyright (C) American Institute of Aeronautics and Astronautics, Inc., 1978. All rights reserved.

Index categories: Nonsteady Aerodynamics; Nozzle and Channel Flow; Transonic Flow.

"Presently, School of Mechanical Engineering, Purdue University, West Lafayette, Ind. Member AIAA.

TProfessor of Aerospace Engineering. Associate Fellow AIAA. downstream of the region under consideration. A more detailed description of the analysis which follows may be found in Ref. 7.

\section{Channel Flow Solutions}

The solution is written in terms of time-dependent perturbations from a steady, uniform, irrotational, sonic flow. The coordinate system and notation used are illustrated in Fig. 1. Velocity components $u$ and $v$ and the sound speed $a$ are made dimensioniess with respect to $\bar{a}^{*}$, the sound speed in the undisturbed flow (overbars indicate dimensional quantities); space variables $x$ and $y$ are referred to $\bar{L}_{8}$ the channel half width at the minimum area; and time $T$ is made dimensionless with respect to $\bar{L} / \bar{a}^{*}$. The pressure, density, and temperature, denoted by $P, \rho$, and $\hat{T}$, respectively, are made dimensionless with respect to their values in the undisturbed flow. The region being considered is that for which $x=O(1)$ and $y=O(1)$; that is, it extends downstream of the throat for distances of the order of the channel half-width. We introduce a small parameter, $\epsilon \ll l$, which is of the order of the typical percentage difference between the flow velocity and its critical sonic value. Thus, $u-1=O(\epsilon)$ for $x=O(1)$. The flow entering the region under consideration is irrotational and boundary-layer effects are negligible. Moreover, because the flow is transonic, shock waves are weak enough that it can be shown ${ }^{7}$ that one can define a velocity potential to the order considered here.

Unsteady flows may be characterized by prescribing the relative orders of the characteristic time associated with the imposed flow disturbance, $\bar{T}_{c h}$, and the characteristic flow time, $\bar{L} / \bar{a}^{*}$. In this present work, the so-called slowly-varying time regime is considered, where $\bar{T}_{c h} \gg \bar{L} / \bar{a}^{*}$. Hence, a parameter $\tau$ is introduced as follows,

$$
\begin{gathered}
T=\tau t \\
\tau=\bar{T}_{c h} /\left(\bar{L} / \bar{a}^{*}\right)
\end{gathered}
$$

where $\tau \gg 1$ and $t=O(1)$. The slowly varying time regime covers a range of values for $\tilde{T}_{c h}$ which are found in many physical problems. ${ }^{6}$ The relationship between $t$ and $\epsilon$ depends upon the relative orders of $\bar{T}_{c h}$ and the time it takes a pilse to move upstream from the point at which a disturbance originates to the location under consideration, e.g., the point at which a shock wave is located. Thus, a disturbance pulse travels upstream at sonic velocity relative to the flow and, therefore, at an absolute velocity $u_{p}=a-u=O(\epsilon)$. Hence, the time required for the disturbance to travel a distance $\bar{L}$ is $O\left(\bar{L} / \epsilon \bar{a}^{*}\right)$, and the desired time ratio is $\bar{T}_{c h} /\left(\bar{L} / \epsilon \bar{a}^{*}\right)=\tau / \epsilon$. It is seen, therefore, that if the two times are of the same order, 


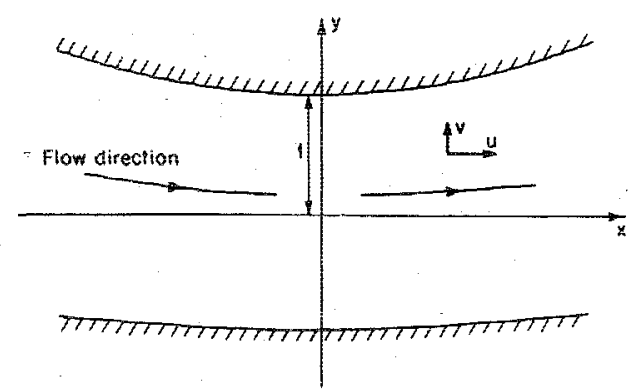

Fig. 1 Sketch of asymmetric channel flow, showing coordinate system.

$t=O(\epsilon)$. This is the case considered in Ref. 6 . Here, we consider the case where $\bar{T}_{c h} \geqslant \bar{L} /\left(\epsilon \bar{a}^{*}\right)$, and, in fact, define $\tau$ as

$$
\tau=\left(k \epsilon^{2}\right)^{-1}
$$

where $k$ is a constant of order unity. Physically, this means that flow disturbances are communicated "instantaneously" to the shock wave.

The governing equations are the gas dynamic equation and the Bernoulli equation:

$$
\begin{aligned}
& \left(a^{2}-\Phi_{x}^{2}\right) \Phi_{x x}+\left(a^{2}-\Phi_{y}^{2}\right) \Phi_{y y}-2 \Phi_{x} \Phi_{y} \Phi_{x y}-\Phi_{T T} \\
& \quad-2 \Phi_{x} \Phi_{x T}-2 \Phi_{y} \Phi_{y T}=0 \\
& \Phi_{T}+1 / 2\left(\Phi_{x}^{2}+\Phi_{y}^{2}\right)+a^{2} /(\gamma-1)=(\gamma+1) / 2(\gamma-I)
\end{aligned}
$$

where subscripts $x, y$, and $T$ indicate partial differentiation. It can be shown ${ }^{7}$ that the constant on the right-hand side of $E q$. (3b) is valid to and including terms of order $\epsilon^{2}$, the highestorder terms needed here. Since the solutions are written in terms of perturbations from a uniform flow, it is convenient to write as

$$
\Phi(x, y, T)=x+\phi(x, y, T)
$$

where $\phi$ is the perturbation potential. Then, using Eq. (3b) for $a^{2}$ and Eq. (4) for $\bar{\varphi}, \mathrm{Eq}$. (3a) may be written as:

$$
\begin{aligned}
\phi_{y y} & \left.=(\gamma+1) \llbracket\left(\phi_{x}+\phi_{x}^{2} / 2\right) \phi_{x x}+\left(\phi_{y}^{z} / 2\right) \phi_{y y}\right] \\
& +2\left(\phi_{y}+\phi_{x} \phi_{y}\right) \phi_{x y}+(\gamma-I)\left[\left(\phi_{x}+\phi_{x}^{2} / 2\right) \phi_{y y}+\left(\phi_{y}^{2} / 2\right) \phi_{x x}\right] \\
& +2\left(I \frac{1}{3} \phi_{x}\right) \phi_{x T}+(\gamma-I) \phi_{T}\left(\phi_{x x}+\phi_{y y}\right)+2 \phi_{y} \phi_{y T}+\phi_{T T}
\end{aligned}
$$

The boundary conditions at the walls of the channel are, for stationary walls,

$$
\phi_{y}\left(x, y_{w}, T\right)=y_{w}^{\prime}\left[1+\phi_{x}\left(x, y_{w}, T\right)\right]
$$

where $y_{w}^{\prime}=\mathrm{d} y_{w} / \mathrm{d} x$. For the asymmetric channels under consideration, and with the coordinate system as shown in Fig. 1 , the wall shape $y_{w}$ is written as

$$
y_{w}= \pm I+\epsilon f_{1}(x) \pm \epsilon^{2} f_{2}(x)
$$

where $f_{l}(0)=f_{2}(0)=f_{I}^{\prime}(0)=f_{2}^{\prime}(0)=0$, and where $f_{i}^{\prime \prime}(x)$ and $f_{2}^{\prime \prime}(x)$ are continuous and nonzero at $x=0$. The upper and lower signs refer to the upper and lower walls, respectively. It is seen that $y=\epsilon f_{i}(x)$ is the equation for the channel centerline and $\pm\left(1+\epsilon^{2} f_{2}(x)\right)$ are terms which describe walls symmetric with respect to the centerline, so that $\epsilon$ is a measure of the radius of curvature of the channel at $x=0$, the channel throat. Thus, the channel curvature is an order of magnitude larger than that considered previously, ${ }^{6}$ and as will be seen, this leads to completely different flow structures and shock shapes.
An asymptotic expansion of $\phi$ is written as follows:

$$
\phi(x, y, T)=\epsilon \phi_{1}(x, y, t)+\epsilon^{2} \phi_{2}(x, y, t)+\epsilon^{3} \phi_{3}\left(x, y_{3} t\right)+\ldots
$$

Substitution of Eqs. (1, 2, and 8) into Eq. (5) yields the following equations for $\phi_{1}, \phi_{2}$, and $\phi_{3}$ :

$$
\begin{gathered}
\phi_{1 y y}=0 \\
\phi_{2 y y}=\left[(\gamma+I) \phi_{l x}^{2} / 2+\phi_{l y}^{2}\right]_{x} \\
\phi_{3 y y}=\left[(\gamma+1) \phi_{I x} \phi_{2 x}+2\left(\phi_{l y} \phi_{2 y}+k \phi_{l t}\right)\right. \\
\left.+1 / 3(\gamma-1 / 2)(\gamma+I) \phi_{I x}^{3}+\gamma \phi_{l x} \phi_{l y}^{2}\right]_{x}-(\gamma+1) \phi_{l y}^{2} \phi_{I x x} / 2
\end{gathered}
$$

The boundary conditions for Eqs. (9) are found by substituting Eqs. (1, 2, 7, and 8) into Eq. (6). Thus, to first order, it is found that

$$
\phi_{l y}(x, \pm 1, t)=f_{I}^{\prime}(x)
$$

where the prime indicates differentiation with respect to $x$. The solution to Eq. (9a), subject to the boundary conditions in Eq. (10), is

$$
\phi_{1}=f_{I}^{\prime}(x) y+h_{1}(x, t)
$$

where $h_{l}(x, t)$ is a function of integration. If the solution for $\phi_{I}$, Eq. (11), is substituted into $\mathrm{Eq}$. (9b), the resulting equation may be integrated to give a solution for $\phi_{2}$. Thus,

$$
\begin{aligned}
& \phi_{2}(x, y, t)=[(\gamma+I) / 12] f_{!}^{\prime \prime} f_{I}^{\prime \prime} y^{4} \\
& \quad+[(\gamma+I) / 6]\left(f_{I}^{\prime \prime} h_{l x}\right)_{x} y^{3}+1 / 2\left(f_{I}^{\prime}\right)^{2} \\
& \left.\quad+[(\gamma+I) / 2]\left(h_{I x}\right)^{2}\right)_{x} y^{2}+g_{2}(x, t) y+h_{2}(x, t)
\end{aligned}
$$

where $g_{2}$ and $h_{2}$ are functions of integration. If the solutions for $\phi_{1}$ and $\phi_{2}$ are substituted into the boundary condition, Eq. (6), where it should be noted that evaluation of any function at $y_{w}$ implies expansion using Eq. (7), then one finds equations for $g_{2}$ and $h_{\ell x}$. Thus,

$$
\begin{gathered}
g_{2}(x, t)=f^{\prime} h_{l x}-[(\gamma+I) / 2]\left(f_{I}^{\prime \prime} h_{I x}\right)_{x} \\
h_{l x}= \pm\left(\left(2 f_{2}-\left(f_{I}^{\prime}\right)^{2}\right) /(\gamma+I)+\beta_{I}(t)-\left(f_{l}^{\prime \prime}\right)^{2} / 3\right\}^{1 / 2}
\end{gathered}
$$

where the equation for $h_{i x}$ has been integrated once, and $\beta(t)$ is the resulting function of integration; $f_{1}$ and $f_{2}$ are both functions of $x$ and defined in Eq. (7). It is seen from Eq. (12) that, in order to complete the solutions to second order, it is necessary to find $h_{2}(x, t)$. This is accomplished by substituting the solutions for $\phi_{1}$ and $\phi_{2}$ into Eq. (9c), integrating once to obtain $\phi_{3 y}$, and substituting the resulting equations into the boundary condition, Eq. (6). One finds that

$$
\begin{aligned}
h_{2 x} & =7(\gamma+I)\left[\left(f_{l}^{\prime \prime}\right)^{2}\right]^{\prime \prime} / 360+\left(f_{I}^{\prime} f_{I}^{\prime \prime \prime}-f_{2}^{\prime \prime}\right) / 6-\left(f_{I}^{\prime}\right)^{2} / 2-f_{I} f_{I}^{\prime \prime} \\
& +\left[(3 / 2-\gamma) h_{I x}^{2}-\gamma\left(f_{l}^{\prime \prime}\right)^{2}\right] / 3+\left[(2(\gamma+l) / 15) f_{i}^{\prime \prime}\left(f_{l}^{\prime \prime} h_{l x}\right)_{x x}\right. \\
& \left.-(2 k /(\gamma+I)) h_{l t}+\beta_{2}(t)\right] / h_{l x}
\end{aligned}
$$

where $\beta_{2}(t)$ is, again, the function of integration. Finally, then, the solutions for $u$ and $v$ are, to second order,

$$
\begin{aligned}
u & =\Phi_{x}=1+\epsilon\left\{f_{l}^{\prime \prime} y+h_{i x}\right\}+\epsilon^{2}\left\{(\gamma+I)\left[\left(f_{l}^{\prime \prime}\right)^{2}\right]^{\prime \prime} y^{4} / 24\right. \\
& +(\gamma+I)\left(f_{l}^{\prime \prime} h_{l x}\right)_{x x} y^{3} / 6+\left(\left(f_{l}^{\prime}\right)^{2}+((\gamma+I) / 2) h_{l x}^{2}\right)_{x} y^{2} / 2 \\
& \left.+\left(f_{l}^{\prime} h_{i x}-[(\gamma+I) / 2]\left(f_{l}^{\prime \prime} h_{i x}\right)_{x}\right)_{x} y+h_{2 x}\right\}+\ldots
\end{aligned}
$$



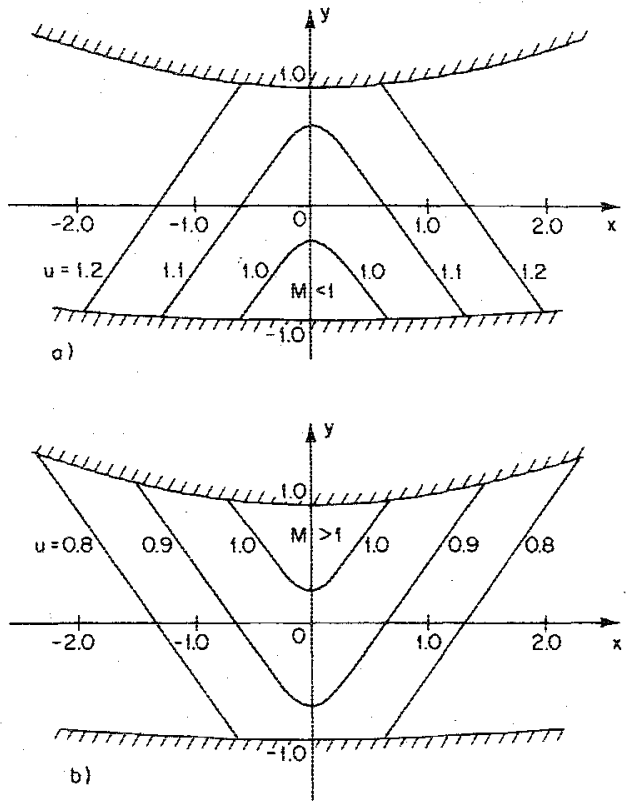

Fig. 2 Isotachs corresponding to Eq. (15a) when $\beta_{l}>\left(f_{l}^{\prime \prime}\right)^{2} / 3 ; y_{w}=$ $\pm 1+\epsilon x^{2} / 2 \pm \epsilon^{2}[1+20(\gamma+1) / 9] x^{2} / 2, \beta_{i}=5 / 12, \epsilon=0.1$. a) + sign taken for all $x$ in Eq. (13b), b) - sign taken for all $x$ in Eq. (13b).
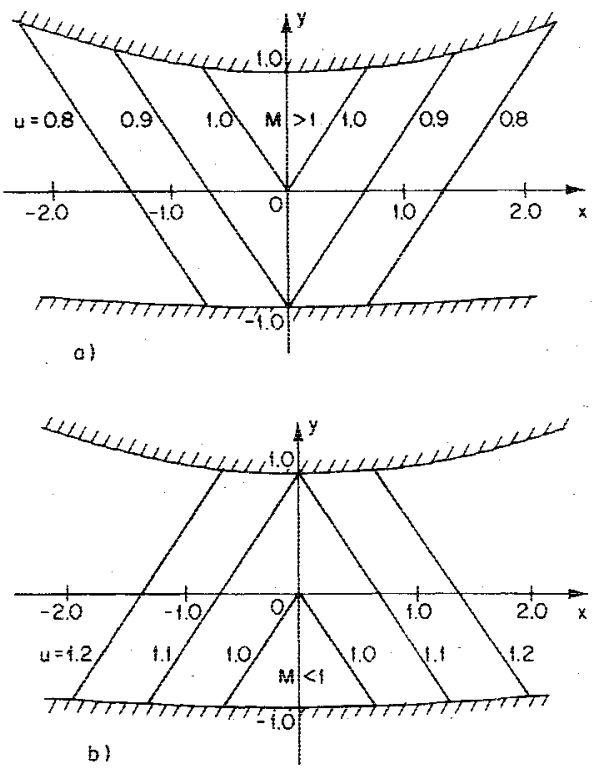

Fig. 3 Isotachs corresponding to Eq. (15a) when $\beta_{I}=\left(f_{I}^{\prime \prime}\right)^{2} / 3=1 / 3$, for some wall shapes and $c$ as in Fig. 2. a) $+\operatorname{sign}$ for $x<0$ and $-\operatorname{sign}$ for $x>0$ in Ea. (13b), b) - sign for $x<0$ and $+\operatorname{sign}$ for $x>0$ in Eq. (13).

$$
\begin{aligned}
v & =\Phi_{y}=\epsilon f_{l}^{\prime}+\epsilon^{2}\left[(\gamma+l)\left[\left(f_{l}^{\prime \prime}\right)^{2}\right]^{\prime} y^{3} / 6\right. \\
& \left.+(\gamma+I)\left(f_{l}^{\prime \prime} h_{l x}\right)_{x} y^{2} / 2+\left(f_{l}^{\prime}\right)^{2}+((\gamma+1) / 2) h_{l x}^{2}\right)_{x} y \\
& +f_{l}^{\prime} h_{l x}-[(\gamma+1) / 2]\left(f_{i}^{\prime \prime} h_{l x}\right)_{x} 1+\ldots
\end{aligned}
$$

where $h_{I x}$ and $h_{2 x}$ are given by Eqs. (13b) and (14), respectively, and $\beta_{1}$ and $\beta_{2}$ are to be determined from the initial conditions of the problem under consideration.

Before proceeding to the consideration of flows with shock waves, it is of interest to establish the range of flow patterns covered by the solutions represented by Eqs. (15). First, it should be noted that in $h_{i x}[\mathrm{Eq} .(13 \mathrm{~b})]$ the term $\beta_{1}-\left(f_{2}^{\prime \prime}\right)^{2} / 3$ cannot be negative, since the remaining terms under the square root are zero at $x=0$. If we first consider the case where $\beta_{1}-\left(f_{l}^{\prime \prime}\right)^{2} / 3>0$, and also consider the solution for $u$
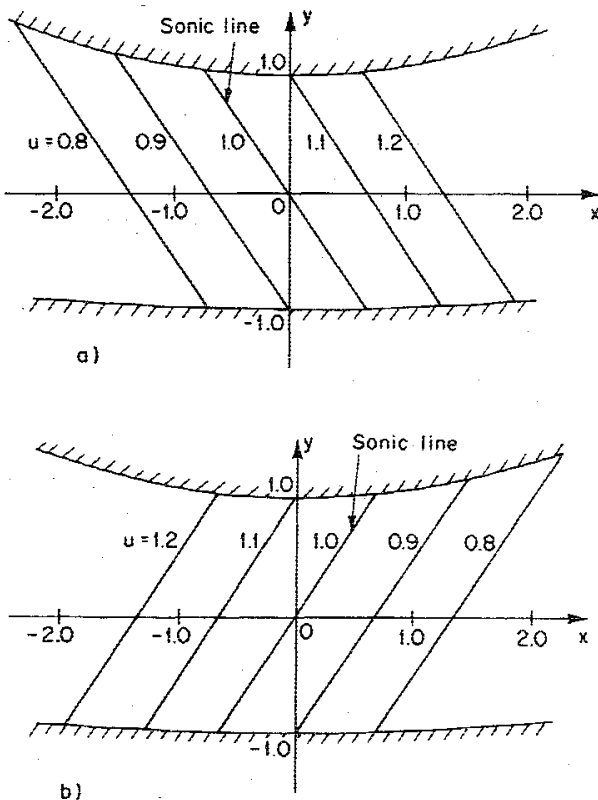

Fig. 4 Isotachs corresponding to Eq. (15a) when $\beta_{1}=\left(f_{i}^{\prime \prime}\right)^{2} / 3=1 / 3$, for same wall shapes and $\epsilon$ as in Fig. 2, a) $+\operatorname{sign}$ taken for all $x$ in Eq. (13b), b) - sign taken for all $x$ in Eq. (13b).

[Eq. (15a)] only up to terms of order $\epsilon$, then if the upper $(+)$ sign is chosen for all $x$ in Eq. (13b), the resulting flow is supersonic, except for a possible subsonic pocket existing in a region around $x=0$, as shown in Fig. 2a. If, on the other hand, the lower ( - ) sign is chosen for all $x$ in Eq. (13b), one obtains a subsonic flow, except for a possible supersonic pocket around $x=0$, as shown in Fig. $2 \mathrm{~b}$. In both cases, the pocket enclosed by the sonic line grows as $\beta_{l}-\left(f_{l}^{\prime \prime}\right)^{2} / 3$ decreases. Until $\beta_{l}-\left(f_{l}^{\prime \prime}\right)^{2} / 3=0$, no sign change at $x=0$ can be tolerated, because the sonic line $(u=1)$ does not pass through $(0,0)$. When $\beta_{1}-\left(f_{i}^{\prime \prime}\right)^{2} / 3=0$, depending upon whether the sign in Eq. (13b) changes or remains unchanged as $x$ passes through zero, the sonic line either returns to the same wall (Figs. $3 a$ and $3 b$ ) or crosses the channel to the opposite wall (Figs. $4 a$ and $4 b$ ). The gradients in velocity, and thus in pressure, temperature, etc., seen in these flow pictures illustrate the very interesting changes which take place in the flow when a radius of channel curvature of order $\epsilon^{-1}$ is considered, as opposed to the radius of curvature of order $\epsilon^{-2}$ considered in Ref. 6 .

It can be shown ${ }^{7}$ that for $x=O(\epsilon)$, the terms of order $\epsilon$ and $\epsilon^{2}$ in $u$ may become of the same order, casting doubt upon the validity of the asymptotic expansions in this region. However, as shown in Refs. 4 and 5, this possible lack of uniform validity for the asymptotic expansions is not a serious problem, the difficulty disappearing, for example, for walls which vary as $x^{2}$ in the region where $x=O(\epsilon)$. Hence, the throat region will not be considered in this paper.

The solutions presented thus far were derived for nows with no shock waves. It is of interest now to analyze cases where shock waves exist and to ascertain if and at what order these solutions break down. We choose, for illustrative purposes, those flows for which $\beta_{I}-\left(f_{l}^{\prime \prime}\right)^{2} / 3=0$, shown in Figs. $3 b$ and $4 \mathrm{a}$. In these cases, in Eq. (13b), the upper $(+)$ sign (supersonic flow) is used downstream of the throat but upstream of the shock, with the lower (-) sign (subsonic flows) holding downstream of the shock; the sign upstream of the throat depends upon whether the flow is subsonic (accelerating, Fig. 4a) or supersonic (decelerating, Fig. 3b). Similar results hold for other sign combinations.

If the shock position is, to lowest order, $x_{s}=x_{0}(t)+\ldots$ then from Eqs. (1a) and (2), it is seen that the shock velocity $\mathrm{d} x_{s} / \mathrm{d} T$ is of order $\epsilon^{2}$. Hence, to order $\epsilon$, the shock polar equation, which reduces to the Prandtl relation, may be 
written in terms of absolute velocities. Thus,

$$
u_{l d}=-u_{l u}
$$

where $u_{1}=\phi_{1 x}$, and the subscripts $u$ and $d$ denote values immediately upstream and downstream of the shock wave, respectively. Now, if Eqs. (11) and (13b) are used to write $u_{f u}$ and $u_{i d}$ at $x_{0}$, where the upper sign in Eq. (13b) is used for $u_{l u}$ and the lower for $u_{I d}$, it is seen that the shock wave jump condition, Eq. (16), is not satisfied. Thus, even in first order, the shock wave jump conditions are not satisfied, as opposed to the case for flows with smaller curvature, ${ }^{6}$ and an inner region about the shock must be considered.

\section{Solutions in the Shock Wave Region}

In the inner region containing the shock wave, the solutions must satisfy the jump conditions across the shock, and match with the outer channel flow solutions in the appropriate limit. This region may be expected to extend across the channel, but to be very thin in the flow direction. Then, in the inner region, we define the independent variables as

$$
\begin{gathered}
x^{*}=\left(x-x_{0}(t)\right) /(\gamma+I)^{1 / 2} \epsilon^{\sigma} \\
y^{*}=\mathrm{y} \\
T^{*}=T=\tau t^{*}
\end{gathered}
$$

where, again, $x_{0}(t)$ is the shock location to lowest order and $\sigma$ is to be determined. The inner region velocity potential is defined as

$$
\Phi^{*}\left(x^{*}, y^{*}, T^{*}\right)=\Phi(x, y, T) /(\gamma+I)^{1 / 2} \epsilon^{\sigma}-\dot{x}_{0} x^{*}
$$

where $\dot{x}_{0}=\mathrm{d} x_{0} / \mathrm{d} T=k \epsilon^{2} \mathrm{~d} x_{0} / \mathrm{d} t$. That is,

$$
\begin{gathered}
u^{*}=\Phi_{x^{*}}^{*}=u-\dot{x}_{0} \\
v^{*}=\Phi_{y^{*}}^{*}=v /(\gamma+l)^{i / 2} \epsilon^{\sigma}
\end{gathered}
$$

In view of the expansion for $\Phi, \mathrm{Eq}$. (4), we write the expansion for $\Phi^{*}$ as follows:

$$
\Phi^{*}\left(x^{*}, y^{*}, T^{*}\right)=\left(I-\dot{x}_{0}\right) x^{*}+\phi^{*}\left(x^{*}, y^{*}, T^{*}\right)+x_{0} /(\gamma+I)^{1 / 2} \epsilon^{\sigma}
$$

where $\phi^{*}\left(x^{*}, y^{*}, T^{*}\right)$ is the inner perturbation potential.

The proper form for the expansion of $\phi^{*}$ results from the application of three conditions which must be fulfilled. Since it is expected that the fluid acceleration in the inner region is of importance and will be found in the lowest-order governing equation for $\phi^{*}$, in order that changes introduced by the shock may be accommodated, the first condition is that $\phi_{I x^{*}}^{*} \phi_{I x^{*} x^{*}}^{*}$ be of the same order as $\phi_{I y^{*} y^{*}}^{*}$. The next condition is given by the matching conditions which are to be met as $\left|x^{*}\right| \rightarrow \infty$. They are found by expanding the outer solutions, Eqs. (15), about $x=x_{0}$ and substituting the resulting equations written in terms of inner variables into Eqs. (19). The final condition is imposed by the boundary conditions at the wall. Since $y-y_{w}=0$ at the walls, then also the Eulerian derivative of this function, $D\left(y-y_{w}\right) / D D T=0$ at the walls. This equation, which may be used to derive Eq. (6), is transformed using Eqs. $(17,19,20)$ and Eq. (7) expanded about $x_{0}$, such that it is valid in the moving coordinate system associated with the inner region, and gives the desired boundary conditions. The three conditions lead to $\sigma=1 / 2$ in Eq. (17a), and

$$
\begin{aligned}
& \phi^{*}\left(x^{*}, y^{*}, T^{*}\right)=\epsilon^{1 / 2} f_{10}^{\prime} y^{*} /(\gamma+I)^{1 / 2} \\
& \quad+\epsilon \phi_{J}^{*}\left(x^{*}, y^{*}, t^{*}\right)+\epsilon^{3 / 2} \phi_{3 / 2}^{*}\left(x^{*}, y^{*}, t^{*}\right)+\ldots
\end{aligned}
$$

where $f_{10}^{\prime}=f_{i}^{\prime}\left(x_{0}\right)$.
The governing equation for $\phi_{I}^{*}$ in the inner region may be derived from Eq. (5) by using Eqs. $(4,18,20,21$, and 17) with $\sigma=1 / 2$. Thus,

$$
\phi_{1 x^{*}}^{*} \phi_{I x^{*} x^{*}}^{*}-\phi_{l y^{*} y^{*}}^{*}=0
$$

The matching conditions to be used as $\left|x^{*}\right| \rightarrow \infty$, found as described previously, are

$$
\begin{gathered}
\phi_{l x^{*}}^{*}=f_{10}^{\prime \prime} y^{*} \pm h_{1 x}\left(x_{0}\right) \\
\phi_{1 y^{*}}^{*}=f_{10}^{\prime \prime} x^{*}
\end{gathered}
$$

where $h_{l x}\left(x_{0}\right)$ is given by Eq. (13b) with the upper $(+)$ sign, and where the upper and lower signs in Eq. (23) correspond to the conditions as $x^{*} \rightarrow-\infty$ and $x^{*} \rightarrow+\infty$, respectively. The boundary conditions at the wall, again found as described previously, are

$$
\phi^{*}{ }_{1 y^{*}}\left(x^{*}, \pm 1, t^{*}\right)=f_{10}^{\prime \prime} x^{*}
$$

The final condition necessary before solutions in the inner region may be found are the shock wave jump conditions. Now, the shape of the shock, $x_{s}^{*}\left(y^{*}, t^{*}\right)$, is given in the inner region by

$$
\frac{\partial x_{s}^{*}}{\partial y^{*}}=-\frac{v_{d}^{*}-v_{u}^{*}}{u_{d}^{*}-u_{u}^{*}}
$$

In view of Eq. (21), it is seen that the first term in the expression for $x_{s}^{*}$ is of order of unity and the next is of order $\epsilon^{1 / 2}$. In the inner and outer variables, then, the expression

$$
\frac{x_{s}(y, t)-x_{0}}{\epsilon^{1 / 2}(\gamma+1)^{1 / 2}}=x_{s}^{*}\left(y^{*}, t^{*}\right)=x_{I}\left(y^{*}, t^{*}\right)+\epsilon^{1 / 2} x_{2}\left(y^{*}, t^{*}\right)+\ldots
$$

where $x_{l}\left(y^{*}, t^{*}\right)=x_{l}(y, t)$, etc., meets the necessary conditions and agrees with the relation used previously that $x_{s}=x_{0}+\ldots$ The function $x_{1}$ is chosen such that $x_{I}(-1, t)=0$; that is, $x_{0}$ gives the position of the intersection of the shock wave and the lower wall, and $x_{l}$ gives the variation of the shock shape from the normal to the $x$ axis at that point. In order to find the jump conditions across the shock wave, it is necessary to write the shock polar equation relative to the moving shock. The velocity components relative to the shock wave are $\hat{u}=u-u_{s h}$ and $\hat{v}=v-v_{s h}$, where $u_{s h}$ and $v_{s h}$ are the components of the shock wave velocity. It can be shown ${ }^{7}$ that $u_{s h}=\dot{x}_{0}+O\left(\epsilon^{5 / 2}\right)$ and $v_{s h}=O\left(\epsilon^{5 / 2}\right)$, and since $\dot{x}_{0}=\epsilon^{2} k \mathrm{~d} x_{0} /$ $\mathrm{d} t=O\left(\epsilon^{2}\right)$, it is seen that if $u_{i}^{*}=\phi_{l x^{*}}^{*}, v_{l}^{*}=\phi_{l y^{*}}^{*}$, etc., then

$$
\begin{gathered}
\hat{u}=l+\epsilon u_{l}^{*}+\epsilon^{3 / 2} u_{3 / 2}^{*}+\ldots \\
\hat{v}=(\gamma+l)^{1 / 2}\left(\epsilon v_{l / 2}^{*}+\epsilon^{3 / 2} v_{l}^{*}+\ldots\right)
\end{gathered}
$$

That is, to the order desired, the shock wave velocity does not appear. Finally, from the energy equation which holds in a moving coordinate system, it can be shown that ${ }^{7}$ the dimensionless critical speed of sound is, in this system,

$$
\hat{a}^{* 2}=I-2(\gamma-1) \dot{x}_{0} /(\gamma+1)+\ldots
$$

The jump conditions are found, then, by expanding Eqs. (27) about $x_{s}^{*}$ using Eqs. (26), and substituting these expansions and Eq. (28) with $\dot{x}_{0}=\epsilon^{2} k \mathrm{~d} x_{0} / \mathrm{d} t$ into the shock polar equation. The results are ${ }^{7}$

$$
\left(v_{l d}^{*}-v_{l u}^{*}\right)^{2}-1 / 2\left(u_{l u}^{*}-u_{l d}^{*}\right)^{2}\left(u_{l u}^{*}+u_{l d}^{*}\right)=0
$$

It may be noted that the form of expansion derived for $u^{*}$ and $v^{*}$, using boundary and matching conditions, is also consistent with the shock jump conditions. 
It is not difficult to show ${ }^{7}$ that upstream of the shock wave, the solutions for $\phi_{l}^{*}$ are simply the continuation of the outer solutions evaluated at the shock wave. That is, one obtains the physically correct result that no disturbances from the shock affect the supersonic flow upstream of the shock wave. Downstream of the shock, however, Eq. (22) must be solved with the boundary conditions given by the condition at the walls, Eq. (24), the matching condition as $x^{*} \rightarrow \infty$, Eq. (23a), and the conditions immediately downstream of the shock wave, given by Eqs. (25) and (29). Thus, since both $u_{i d}^{*}$ and $v_{l d}^{*}$ are found in the first-order jump condition given by $\mathrm{Eq}$. (29), the shock wave is not planar to this order, and another equation, Eq. (25), is necessary to relate the local shock slope to $v_{l d}^{*}$ and $u_{l d}^{*}$. It may be noted that this problem is, therefore, quite different from those solved previously for channels with smaller curvature, ${ }^{6}$ where the shock is planar to the lowest order. In that case, the boundaries and normal derivatives of the potential at the boundaries were all given, and $v_{l d}^{*}$ was calculated, as was the higher-order shock shape. In the present case, however, $u_{l d}^{*}$ and $v_{l d}^{*}$ are related to the shock shape, which must be found as part of the first-order solution.

It is convenient to write $\phi_{I}^{*}$ in terms of the difference between it an its limiting value as $x^{*} \rightarrow \infty$. Thus, we define $\zeta^{*}$ as

$$
\zeta^{*}=\phi_{I}^{*}-\left[f_{I 0}^{\prime \prime} y^{*}-h_{I x}\left(x_{0}\right)\right] x^{*}
$$

Then the governing equation and boundary conditions for the problem to be solved are:

$$
\begin{gathered}
\left(f_{10}^{\prime \prime} y^{*}-h_{l x}\left(x_{0}\right)+\zeta_{x^{*}}^{*}\right) \zeta_{x^{*} x^{*}}^{*}-\zeta_{y^{*} y^{*}}^{*}=0 \\
x^{*}=x_{I} \quad \zeta_{x^{*}}^{*}=-2 f_{10}^{\prime \prime} y^{*}+2\left(\partial x_{1} / \partial y\right)^{2} \\
x^{*}=x_{I} \quad \zeta_{y^{*}}^{*}=2\left(\partial x_{1} / \partial y\right)\left[f_{I 0}^{\prime \prime} y^{*}+h_{i x}\left(x_{0}\right)-\left(\partial x_{1} / \partial y\right)^{2}\right] \\
x^{*} \rightarrow \infty \quad \zeta_{x^{*}}^{*}=0 \\
y^{*}= \pm 1 \quad \zeta_{y^{*}}^{*}=0
\end{gathered}
$$

where

$$
\begin{gathered}
\frac{l}{(\gamma+1)^{1 / 2} \epsilon^{1 / 2}} \frac{\partial x_{s}}{\partial y}=\frac{\partial x_{s}^{*}}{\partial y^{*}}=\frac{\partial x_{I}}{\partial y}+O\left(\epsilon^{1 / 2}\right) \\
\frac{\partial x_{I}}{\partial y}=-\frac{v_{l d}^{*}-v_{l u}^{*}}{u_{l d}^{*}-u_{l u}^{*}}
\end{gathered}
$$

and Eqs. (15) evaluated at the shock wave, and Eqs. (19) have been used to write $u_{l u}^{*}$ and $v_{l u}^{*}$. Evidently, this problem must be solved numerically; specific example solutions are discussed later.

A composite solution, uniformly valid throughout the channel, may be constructed by adding the outer and inner solutions and substracting those (matching) terms common to both. In view of the definition of $\zeta^{*}$, these composite solutions are thus, to first order,

$$
\begin{gathered}
u=l+\epsilon\left(f_{I}^{\prime \prime} y \pm h_{I x}+\zeta_{x^{*}}^{*}\right)+\ldots \\
v=\epsilon f_{l}^{\prime}+\epsilon^{3 / 2}(\gamma+I)^{1 / 2} \zeta_{y^{*}}^{*}+\ldots
\end{gathered}
$$

where $h_{l x}$ is given by Eq. (13b) with the plus sign, and $\zeta^{*}=0$ upstream of the shock, $x<x_{s}$. Also, for $x<x_{s}$, if the flow accelerates from subsonic to supersonic flow [with the sonic line passing through $(0,0)$ ], then the upper sign is taken in Eq. (33a); if the flow is supersonic, decelerating flow for $x<0$ and accelerating for $x>0$ with possibly a subsonic pocket in the region about $x=0$, then the lower sign is taken for $x<0$ and the upper for $x>0$. For $x>x_{s}$, only the lower sign in Eq. (33a) is taken. It should be noted that for $x^{*}=O(1)$, Eqs. (33) reduce to the inner solutions downstream of the shock, while as $x^{*} \rightarrow \infty, \zeta^{*} \rightarrow 0$, and the outer solutions, to order $\epsilon$, are recovered.

Solutions for the pressure and density are written in terms of the solutions for the velocity components through equations derived from the conservation equations and the equation for the jump is entropy across the shock wave. Thus, from the inviscid energy equation, it can be shown ${ }^{7}$ that the stagnation or total enthalpy can be written as

$$
\begin{aligned}
& h_{t}=\frac{\hat{T}}{\gamma-1}+\frac{1}{2}\left(u^{2}+v^{2}\right)=\frac{\gamma+1}{2(\gamma-I)}+\epsilon^{3} h_{t l}+\ldots \\
& h_{t I}=k \frac{\mathrm{d} x_{0}}{\mathrm{~d} t}\left(\zeta_{x^{*}}^{*}-2 h_{I x}\left(x_{0}\right)\right) \quad x>x_{s} \\
& h_{t l}=0 \quad x<x_{s}
\end{aligned}
$$

where $h_{I x}\left(x_{0}\right)$ is calculated from Eq. (13b) with the plus sign. The pressure and density are related to the entropy and the temperature as follows:

$$
\begin{gathered}
P / \rho^{\gamma}=e^{\left(s-s_{k}\right)(\hat{z}-1)} \\
P=\rho \hat{T}
\end{gathered}
$$

The entropy change of importance is that across the shock wave, the gradients downstream of the shock being of high enough order that they are negligible. For transonic flow, across a shock ${ }^{8}$

$S_{d}-S_{u}=\frac{2 \gamma}{3(\gamma+I)}\left(M_{u}^{2}-I\right)^{3}-\frac{2 \gamma^{2}}{(\gamma+I)^{3}}\left(M_{u}^{2}-I\right)^{4}+\ldots$

where, since the shock in this case is in motion, $M_{u}$ is the relative normal Mach number of the incoming flow. For a shock described by Eq. (26),

$$
M_{u}^{2}-I=\epsilon(\gamma+l)\left[u_{I u}-\left(\partial x_{I} / \partial y\right)^{2}\right]
$$

where, as mentioned earlier, outer solutions are valid upstream of the shock wave. The expansions for $P$ and $\rho$ can then be found from Eqs. (34a) and (35), where $u$ and $v$ are found from Eqs. (4) and (8) with $u_{i}=\phi_{i x}$ and $v_{i}=\phi_{i y}$. Thus,

$$
\begin{aligned}
P & =I-\epsilon \gamma u_{i}-\epsilon^{2} \gamma\left(v_{I}^{2} / 2+u_{2}\right)-\epsilon^{3} \gamma\left[2 ( \gamma + I ) \left[u_{l t}\right.\right. \\
& \left.-\left(\partial x_{1} / \partial y\right)^{2}\right]^{3} / 3-h_{l I}-(\gamma+I) u_{l}^{3} / 6-u_{l} v_{l}^{2} / 2 \\
& \left.+v_{1} v_{2}+u_{3}\right\}+\ldots \\
\rho & =I-\epsilon u_{l}-\epsilon^{2}\left[(\gamma-1) u_{l}^{2} / 2+v_{l}^{2} / 2+u_{2}\right] \\
& -\epsilon^{3}\left[2 \gamma(\gamma+l)\left(u_{l u}-\left(\partial x_{I} / \partial y\right)^{2}\right)^{3} / 3\right. \\
& -h_{l l}-\gamma(2-\gamma) u_{l}^{3} / 3+(\gamma-1) u_{l} u_{2}-(2-\gamma) u_{l} v_{l}^{2} / 2 \\
& \left.+v_{l} v_{2}+u_{3}\right]+\ldots
\end{aligned}
$$

If the composite solutions are used for $u_{1}$ and $v_{1}$ [terms of order $\epsilon$ in Eqs. (33)], then Eqs. (38) give solutions which are uniformly valid to order $\epsilon$. A solution for $\hat{T}$ similar to those given for $P$ and $\rho$ is easily derived from Eq. (34a).

It should be noted that, if composite solutions are considered, then immediately upstream of the shock wave $P_{y}=-$ $\epsilon \gamma f_{10}^{\prime \prime}$, while downstream of the wave $P_{y}=-\epsilon \gamma\left(f_{10}^{\prime \prime}\right.$ $\left.+\zeta_{x^{*} y^{*}}^{*}\right)+\ldots$ Thus, downstream of the wave, if the wave is approached by first going to $y^{*}= \pm 1$ and then to $x^{*}=x_{1}$, then at the wave $P_{y}=-\epsilon \gamma f_{10}^{\prime \prime}$. However, if the wave is approached by going to $x^{*}=x_{1}$ first at any $y^{*}$, and then traveling along the wave toward $y^{*}= \pm 1, P_{y}=\epsilon \gamma f_{10}^{\prime \prime}+\ldots$. That is, from Eqs. 
(31c) and (3ie), $\partial x_{1} / \partial y=0$ at $y= \pm 1$; hence, from Eq. (3ib), $\zeta_{x^{*} y^{*}}^{*}=-2 f_{10}^{\prime \prime}$ at $y= \pm 1$. Thus, there is a change of sign in $P_{y}$ across the shock, and since $P_{y} \propto v_{I x^{*}}^{*}$, the streamline curvature, this means that downstream of the shock wave, the limiting value of $v_{i x^{*}}^{*}$ at the intersection of the shock wave and the walls depends on the path of approach to the intersection. This singularity in the solution is similar to that analyzed by Messiter and Adamson. ${ }^{5}$

\section{Shock Wave Location}

The unsteady perturbations in flow velocity and thermodynamic properties in the channel can be caused, for example, by pressure disturbances in a plenum downstream of the shock wave. Here, we consider the case where the disturbances have a characteristic time (inverse of characteristic frequency) of order $\epsilon^{-2}$ and an amplitude of order $\epsilon^{2}$. As will be seen, this is sufficient to give shock motions with an amplitude of order unity.

Since only terms of order $\epsilon^{2}$ in $P$ may vary with time, it is seen from Eq. (38a) that $u_{1}$ is independent of time; hence, $h_{l}=h_{l}(x)$. In fact [Eqs. (38a) and (15)], the time dependence first appears in $u_{2}$ through $h_{2}=h_{2}(x, t)$. As a result, in Eq. (13b), $\beta_{1}$ is a constant and the time dependence of $h_{2}$ and thus $u_{2}$ and indeed $\phi_{2}$, is through $\beta_{2}(t)$. Both $\beta_{1}$ and $\beta_{2}$ may have different values upstream and downstream of the shock wave. Since the time enters the solutions for $u$ only through the integration function $\beta_{2}$ then to order $\varepsilon^{2}$, the unsteady motion may be pictured as a sequence of steady-state solutions for $u$, each with different downstream conditions. The velocity component $v$, to order $\epsilon^{2}$, is totally time independent [Eq. (15b)]. From Eqs. (38a, 15, and 14), it is seen that specifying the second-order variation in pressure at a downstream plenum location is equivalent to setting $\beta_{2}(t)$ downstream of the shock, say $\beta_{2 d}$. The fact that time does not appear explicitly in the solutions valid downstream of the shock wave [e.g., see the composite solutions given in $\mathrm{Eq}$. (33)] and appears only as a parameter in $\beta_{2}$ is due to the fact that the disturbances travel upstream at a speed of order $\epsilon$, while the shock moves at a velocity of order $\epsilon^{2}$, and indeed, the order of the unsteady part of the fluid velocity is $\epsilon^{2}$. Thus, the disturbances travel upstream instantaneously, compared with the characteristic times under study. It should be noted, however, that as a result of the motion of the shock li.e., since $\left.x_{0}=x_{0}(t)\right]$ the first-order pressure, velocity, etc., in the range of motion of the shock, do change as the shock moves back and forth; they jump between the steady-state values upstream and downstream of a shock wave at the point in question.

The equation from which the instantaneous shock wave location can be calculated is derived from the principle of mass conservation applied to a control volume enclosing the shock wave. Thus, one surface of the control volume, the sides of which are the channel walls, is located at $x=0$, the throat), and the other at $x=X_{c}$, somewhere downstream of the shock. Then,

$$
\frac{\mathrm{d}}{\mathrm{d} T}\left\{\int_{y_{w}^{-}}^{y_{w}^{+}} \int_{0}^{X_{c}} \rho \mathrm{d} x \mathrm{~d} y\right\}-\int_{-1}^{+1} \rho u \mathrm{~d} y+\int_{y_{w}^{-}\left(X_{c}\right)}^{y_{w}^{+}\left(X_{c}\right)} \rho u \mathrm{~d} y=0
$$

where $y_{w}^{+}$and $y_{w}^{-}$denote, respectively, the upper and lower walls. The integral in the first bracket, from zero to $X_{c}$, is evaluated in two parts, one upstream of the wave $\left(0\right.$ to $\left.x_{s}\right)$ and one downstream of the wave $\left(x_{s}\right.$ to $\left.X_{c}\right)$, with the composite solutions used for $u_{1}$ in the first-order terms of Eq. (38b). Since $d / d T=O\left(\epsilon^{2}\right)$, and only terms up to third order are desired, only the first-order term in $\rho$ is necessary. In the remaining integrals at $x=0$ and $x=X_{c}$, Eqs. (15) and (38b) are used. First-order terms are identically zero, and from the second- and third-order terms, one finds that

$$
\beta_{I d}=\beta_{I d}=\beta_{I}
$$

$$
\begin{aligned}
& \frac{4 k}{(\gamma+1)} \frac{\mathrm{d} x_{0}}{\mathrm{~d} t}=\frac{\beta_{2 u}-\beta_{2 a}}{h_{10}^{\prime}}-2 \gamma \frac{\left(f_{10}^{\prime \prime}\right)^{2}+\left(h_{10}^{\prime}\right)^{2}}{3}+G\left(x_{0}\right) \\
& G\left(x_{0}\right)=\frac{\gamma}{h_{10}^{\prime}} \int_{-1}^{1}\left(\frac{\partial x_{1}}{\partial y}\right)^{2}\left[u_{1 u}^{2}\left(x_{0}\right)\right. \\
& \left.-u_{l u}\left(x_{0}\right)\left(\frac{\partial x_{1}}{\partial y}\right)^{2}+\frac{1}{3}\left(\frac{\partial x_{i}}{\partial y}\right)^{4}\right] \mathrm{d} y
\end{aligned}
$$

where $h_{10}^{\prime}=\mathrm{d} h_{l} / \mathrm{d} x$ evaluated at $x_{0}$ and $\beta_{2 l i}$ is a constant, while $\beta_{2 d}$ is a function of time for the case under consideration, where disturbances are imposed downstream of the shock wave. If the shock wave has a higher-order curvature, $\partial x_{i} / \partial y=0$, and $G\left(x_{0}\right)$ vanishes; then Eq. (40b) is reduced to a form equivalent to that given by Richey and Adamson. ${ }^{6}$

Equation (40b) may be used in determining the shock location, $x_{0}=$ const, for a flow which is steady with a stationary shock wave. Thus, for a given (now constant) $\beta_{2 d}$, which is equivalent to a given pressure in a plenum downstream of the shock wave, the only unknowns in Eq. (40b), $h_{10}^{\prime}$ and $G\left(x_{0}\right)$, are functions of $x_{0}$. Since $\partial x_{I} / \partial y$, which

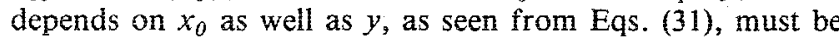
known if $G\left(x_{0}\right)$ is to be calculated, this means that Eqs. (31) and (40) must be solved simultaneously. In principle, therefore, $x_{0}$ may be calculated for a given $\beta_{2 d}$. However, it is much easier to choose a given $x_{0}$, solve Eqs. (31) and in the process find $x_{1}$, calculate $G\left(x_{0}\right)$ and $h_{10}^{\prime}$, and then use Eq. (40b) to calculate the $\beta_{2 d}$ which corresponds to the chosen $x_{0}$. After a series of such calculations, $x_{0}$ may be plotted as a function of $\beta_{2 d}$. If the downstream pressure varies with time in a preseribed manner, then $\beta_{2 d}=\beta_{2 d}(t)$ is known and $\mathrm{d} x_{0} / \mathrm{d} t$ and thus the shock velocity may, in principle, be calculated from Eqs. (31) and (40b), with the shock position, $x_{0}(t)$, being found then by integration of $\mathrm{d} x_{0} / \mathrm{d} t$. The computations may be carried out by first solving Eqs. (31) for a sequence of values for $x_{0}$, so that $\partial x_{1} / \partial y, h_{10}^{\prime}$, and thus $G\left(x_{0}\right)$ are known essentially as functions of $x_{0}$. With $\beta_{2 d}(t)$ and the initial value of $x_{0}$ known, $\mathrm{Eq}$. (40b) may then be integrated numerically, thus relating $x_{0}$ to $t$; i.e., $x_{0}(t)$ is obtained. Finally, with $x_{0}(t)$ known, $\xi^{*}$ and $\partial x_{l} / \partial y$, and thus, $u_{3} P, \rho_{3} x_{i}$, etc, may be obtained as functions of time at any space point.

\section{Numerical Calchlations}

In this paper, Eqs. (31) were solved using the method of integral relations proposed by Dorodnitsyn. ${ }^{9,10}$ Details of the computation are available in Ref. 7 , so only a brief description is given here. The inner region in which Eqs. (31) are valid is composed of a region extending from $y^{*}=-1$ to $y^{*}=+1$ in the $y^{*}$ direction and from the curve $x^{*}=x_{s}^{*}$ to $x^{*} \rightarrow \infty$ in the $x^{*}$ direction; $x_{s}^{*}=x_{1}+\ldots$ is to be found as part of the solution. This region is transformed into a finite region by the transformation $\hat{x}=e^{-x^{*}}$, and this finite region, between $\hat{x}=0$ and $\hat{x}=\hat{x}_{s}=e^{-x_{s}^{*}}$, is divided into $N$ strips. Calculations of the shock shape made with $N=2$, and compared with the calculation made with $N=3$, showed very little difference, indicating that relatively accurate computations can be made with two strips. Equation (31a), written in terms of velocity components, and the corresponding irrotationality condition $u_{i y^{*}}^{*}=v_{/ x^{*}}^{*}$ are the governing equations integrated across each strip, using $N$ th order polynomials in $\hat{x} / \hat{x}_{s}$ for interpolation expressions for $w_{l}^{*}$ and $v_{l}^{*}$. In the present case, it was found that if $\lim _{x \rightarrow \infty} \zeta=0$ is used as a boundary condition and the method of integral relations applied, then $\zeta_{y^{*}}^{*}$ is not zero as $x^{*} \rightarrow \infty$. That is, apparently due to the approximations inherent in the method, and the effects of the singularity at the shock wall interaction, there is an error in the $v$ velocity as $x^{*} \rightarrow \infty$. Rather than accept this error, the condition $\zeta_{y^{*}}^{*} \rightarrow 0$ as $x^{*} \rightarrow \infty$ was enforced and the irrotationality condition was integrated once across the whole region rather than across each of the two strips separately. 


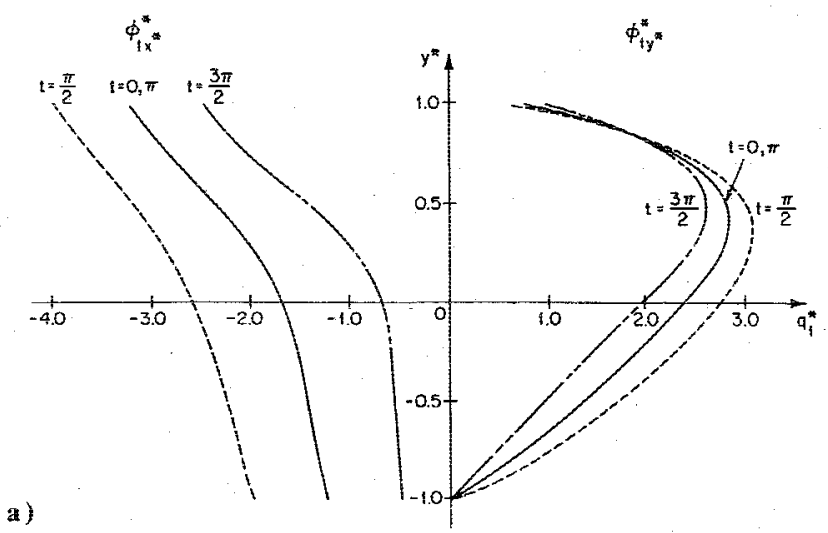

a)

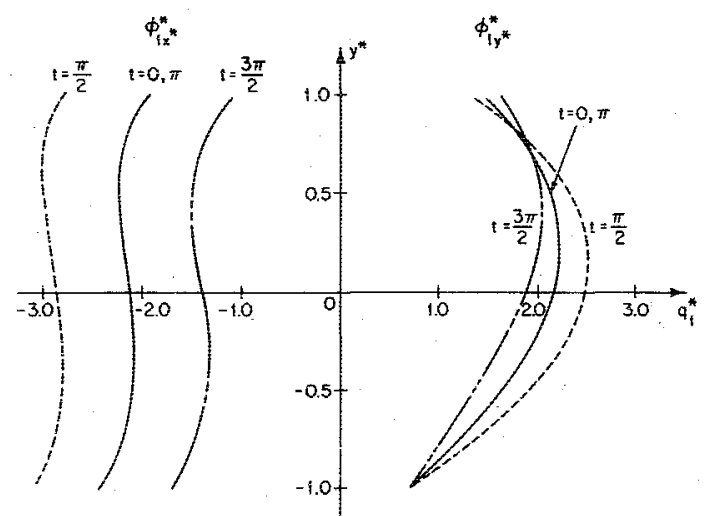

b)

Fig. 5 First-order velocity component profiles downstream of the Shock wave at various times for channel walls given by Eq. (43) and shock location as in Eq. (44), $\gamma=1$.4. a) $x^{*}=x_{1}$, b) $x^{*}=x_{1}+0.69$.

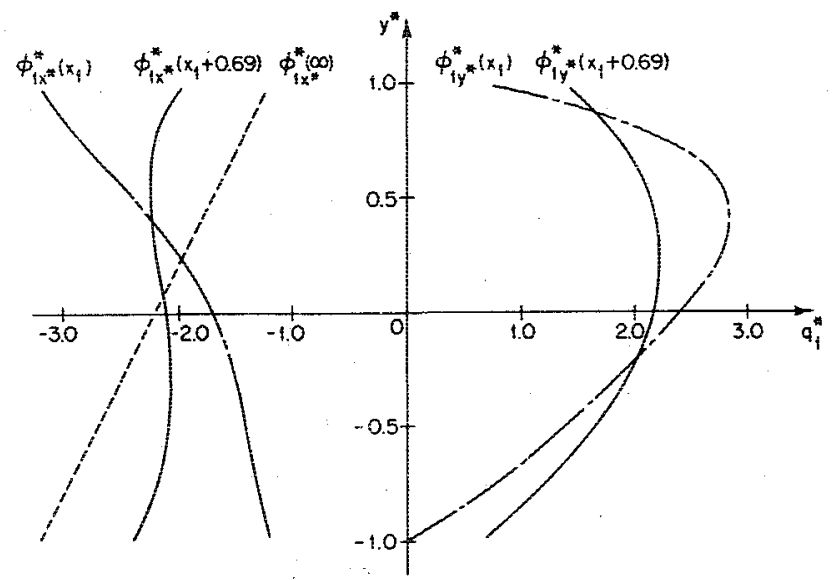

Fig. 6 First-order velocity component profiles at various positions downstream of the shock at $t=0$ for channel walls given by $\mathrm{Eq}$. (43) and shock location as in Eq. (44), $\phi_{l y^{*}}^{*}\left(\infty, y^{*}, 0\right)=0, \gamma=1.4$.

Using the two-strip method, one finds a nonlinear differential equation for the shock shape; since time enters only as a parameter, this equation may be treated as an ordinary differential equation. Thus, if $z(y)=\partial x_{l} / \partial y$, one finds that

$$
\begin{aligned}
\left(f_{10}^{\prime \prime} y+h_{10}^{\prime}-3 z^{2}\right) z^{\prime \prime}-\left[6 z z^{\prime}-2 R z+f_{10}^{\prime \prime} h_{10}^{\prime}\left(1-y^{2}\right)-2 f_{10}^{\prime \prime}\right] z^{\prime} \\
\quad-\left[2 R^{2}+\left(3 h_{10}^{\prime}+2 f_{10}^{\prime \prime} y\right) R-2 f_{10}^{\prime \prime} h_{10}^{\prime} y\right] z \\
\quad-\left[f_{10}^{\prime \prime} h_{10}^{\prime}\left(1-y^{2}\right)+3 f_{10}^{\prime \prime}\right] R-2 f_{10}^{\prime \prime} y-f_{10}^{\prime \prime} h_{10}^{\prime}=0 \\
R=\left[\left(f_{10}^{\prime \prime}\right)^{2} y^{2}+f_{10}^{\prime \prime} h_{10}^{\prime} y+\left(h_{10}^{\prime}\right)^{2}+f_{10}^{\prime \prime} h_{10}\left(1-y^{2}\right) z-f_{10}^{\prime \prime} z\right. \\
\left.-\left(f_{10}^{\prime \prime} y+h_{10}^{\prime}-3 z^{2}\right) z^{\prime}\right]^{1 / 2}
\end{aligned}
$$

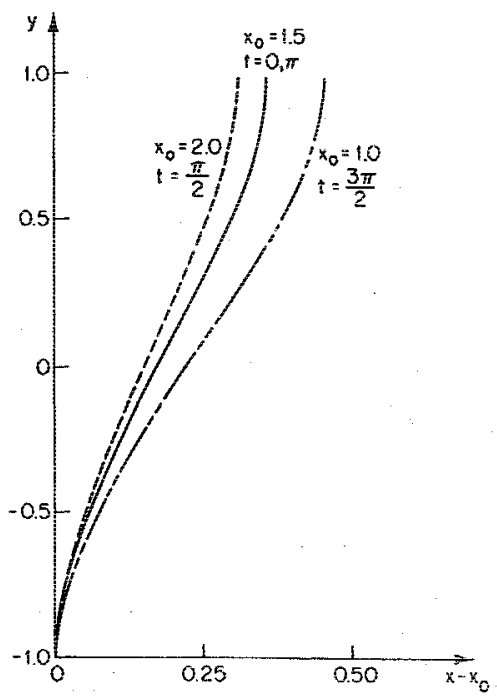

Fig. 7 Variation of shock wave shape with time for chathne wath given by $\mathrm{Eq}$. (43) and shock location as in Eq $(44), \gamma=1,4, \epsilon=0.1$.

where $h_{l 0}^{\prime}=h_{I}^{\prime}\left(x_{0}\right)$, etc. The boundary conditions for Egs. (44) are

$$
z( \pm I)=0
$$

Equation (4la) was solved numerically using a fourth-order Runge-Kutta method, yielding $z(y)$. Then $z(y)=\partial x_{i} / \partial y$ was integrated, using the condition that $x_{l}(-1)=0$, to give the shock shape $x_{1}$. Since both $f_{10}^{\prime \prime}$ and $h_{i 0}^{\prime}$ are time dependert because $x_{0}=x_{0}(t)$, the coefficients in Eqs. (A1) have different values at different times, and it is seen that the shock stape varies with time.

The wall shapes chosen for the calculations are

$$
y_{w}= \pm 1+\epsilon x^{2} / 2 \pm \epsilon^{2}[1+20(\gamma+1) / 9] x^{2} / 2
$$

where the wall shape functions, $f_{1}(x)$ and $f_{2}(x)$ are found by comparing Eqs. (7) and (43). In order to make the calculations as simple as possible, $x_{0}(t)$ is prescribed here and the corresponding $\beta_{2 d}$ (and thus pressure in the dowrotreant plenum) is calculated from Eqs. (40b) and (40c). That is, one can prescribe $\beta_{2 d}(t)$ and find the resulting $x_{\theta}(t)$, or presthe $x_{0}(t)$ and find the necessary $\beta_{2 d}(t)$. The latter problem demands less computing time and is used here for ilnstration. Thus, we set

$$
x_{0}(t)=1.5+(\sin t) / 2
$$

and perform calculations for $\gamma=1.4, \epsilon=0.1$, and $\beta_{2 l i}=0$. Tho wall shapes given by Eqs. (43) with these values for $\epsilon$ and $\gamma$, are those seen in Figs. 2-4.

Velocity profiles showing the first-order inner solutions downstream of the shock wave are shown at $x^{*}=x_{1}$ in Fig. $5 \mathrm{~s}$ and at $x^{*}=x_{1}+0.69$ in Fig. $5 \mathrm{~b}$, each at three different tmes, $t=\pi / 2, \pi$, and $3 \pi / 2$. The corresponding $x_{0}$ is found from $E$. (44). These figures thus illustrate the temporal variations the velocity components at the indicated inner-region stations. The relatively large spatial variations, which occur is the inner region at a given time, are illustrated in Fig. 6 at $i=0$. The change in $u_{i}^{*}$, from its values immediately benind the shock to the linear profile associated with subsonic flow for the given wall shapes, is indicative of the large accelerations and decelerations which take place in the inner region.

The shock shape, $x_{5}=x_{0}+\epsilon^{1 / 2}(\gamma+1)^{1 / 2} x_{1}+\ldots$, is shown Fig. 7 as a function of time. It is seen that, as a result of thit? curvature of the walls and the attendant gradients in the incoming flow, the shock wave has a pronounced curvatme? for the direction of curvature chosen for the walls, the shoc: 

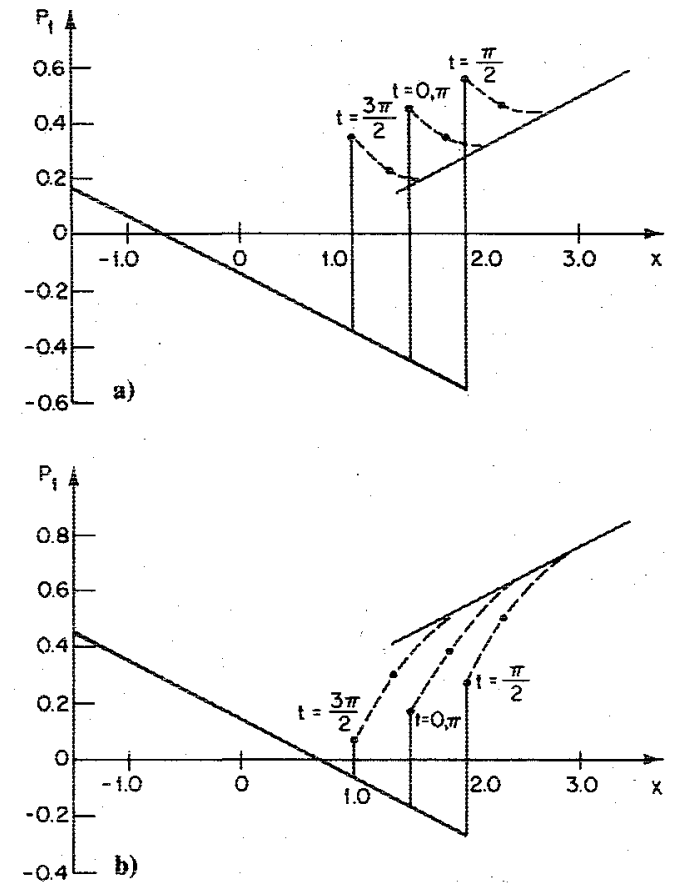

Fig. First-order wall pressure distributions for various times for channel walls given by Eq. (43) and shock location as in Eq. (44), $\gamma=1.4, \epsilon=0.1$. - calculated pressure distribution upstream of the shock wave, jump across shock, and outer pressure distribution downstream of shock; - pressures calculated in inner region; --- approximate pressure distribution in inner region. a) Upper wall, b) Lower wall.

starts normal to the lower wall, inclines in the flow direction, and then turns back toward the upper wall so as to become normal to it also. As the shock moves closer to the throat of the channel, it becomes weaker and has more curvature.

The values of $x_{0}$, for each time at which calculations were made, the corresponding $\beta_{2 d}(t)$ calculated from Eq. (40b), and the equivalent change in back pressure at a downstream plenum from its value at $t=0, \Delta P_{b}=P_{b}(t)-P_{b}(0)$, are given in Table 1. Finally, the pressure on the upper and lower walls, at the same times, to order $\epsilon$, are shown in Fig. 8. In this figure, the solid lines indicate the calculated pressure distributions upstream of the shock wave, the pressure jump across the shock, and outer pressure distributions downstream of the shock. The large dots indicate the pressures calculated in the inner region, and the dashed lines show a curve drawn through these points and faired into the outer pressure distribution, to show an approximate pressure distribution. Since only two strips were used in the innerregion computations, only one data point within the inner region is available.

\section{Conclusions}

The methods used in this study enable one to analyze unsteady flows with shock waves in relatively highly-curved
Table 1 Vaues of $x_{\theta}, P_{2 d}$, and $\Delta F_{b}$ for various times

\begin{tabular}{cccc}
\hline \hline$t$ & $x_{0}$ & $\beta_{2 d}$ & $\Delta P_{b}$ \\
\hline 0 & 1.5 & -12.292 & 0 \\
$\pi / 2$ & 2.0 & -25.708 & 0.1878 \\
$\pi$ & 1.5 & -8.883 & -0.0477 \\
$3 \pi / 2$ & 1.0 & -3.047 & -0.1294 \\
\hline \hline
\end{tabular}

asymmetric channels with arbitrary wall shapes and impressed disturbances of arbitrary form. It is shown that the shock wave is not planar and that its shape must be obtained as part of an inner solution, which involves a numerical solution of the nonlinear small disturbance transonic equation, the unknown shock shape forming one of the boundaries of the region in question. It proves to be relatively easy to use the method of integral relations to obtain approximate but very useful solutions. The solutions allow one to calculate the shock shape and velocity, as well as fluid velocity and thermodynamic property distributions, as function of time.

\section{Acknowledgment}

This work was partially supported by the Office of Naval Research, Project SQUID, under Contract N00014-67-0226005. This support is gratefully acknowledged. The authors wish to thank A. E. Messiter, The Unviersity of Michigan, for many stimulating discussions concerning this work.

\section{References}

'Sichel, M., "The Effect of Longitudinal Viscosity on the Flow at a Nozzle Throat," Journal of Fluid Mechanics, Vol. 25, 1966, pp. 769786.

${ }^{2}$ Adamson Jr., T. C. and Richey, G. K., "Unsteady Transonic Flows with Shock Waves in Two Dimensional Channels," Journal of Fluid Mechanics, Vol. 60, 1973, pp. 363-382.

${ }^{3}$ Kopystynski, J. and Szaniawski, A., "Structure of Flow in a Nozzle Throat," Archiwum Mechaniki Stosowanej, Vol. 17, 1965, pp. 453-466.

${ }^{4}$ Adamson Jr., T. C., Messiter, A. F., and Richey, G. K. "On the Matching of Solutions for Unsteady Transonic Nozzle Flows," Archiwum Mechaniki Stosowanej, Vol. 26, 1974, pp. 617-628.

${ }^{5}$ Messiter, A. F. and Adamson Jr., T. C., "On the Flow Near a Weak Shock Downstream of a Nozzle Throal," Journal of Fluid Mechanics, Vol. 69, 1975, pp. 97-108.

${ }^{6}$ Richey, G. K. and Adamson Jr., T. C., "Analysis of Unsteady Transonic Flow with Shock Waves," AIAA Joumal, Vol. 14, Aug. 1976, pp. 1054-1061.

${ }^{7}$ Chang, J. S.-K., "Unsteady Transonic Flows with Shock Waves in an Asymmetric Channel," PhD Thesis, The University of Michigan, 1976.

${ }^{8}$ Liepmann, H. W. and Roshko, A., Elements of Gas Dynamics, Wiley, New York, 1957, p. 60 .

${ }^{9}$ Dorodnitsyn, A. A., "A Contribution to the Solution of Mixed Problems of Transonic Aerodynamics," Advances in Aeronautical Sciences, Vol. 2, eds., T. Von Kármán, et al., Academic Press, New York, 1965, pp. 832-844.

${ }^{10}$ Belotserkovski, O. M. and Chushkin, P. I., "The Numerical Solution of Problems in Gas Dynamics," Chap. 3, Basic Developments in Fluid Mechanics, Vol. 1, M. Holt, ed., Academic Press, New York, 1965, pp. 34-89. 